\title{
ESTUDIO DE LOS EFECTOS PRODUCIDOS POR LAS CONDICIONES DE TRANSFORMACIÓN EN LA VISCOSIDAD DEL POLIETILENTEREFTALATO (PET) RECICLADO.
}

\section{Study of the effects produced by the transformation conditions on the viscosity of recycled polyethylenterephthalate (pet).}

${ }^{1}$ Julio-Cesar Velázquez-Infante* (D), ${ }^{2}$ Arles Vega García iD, ${ }^{2}$ Rosalia Esquivel Figueredo iD, ${ }^{2}$ Sandra Bárcenas Martínez iD, ${ }^{3}$ Hassan Martínez-Hung iD

${ }^{1}$ Unidad de Extensión, Investigación y Capacitación Agropecuaria de Holguín, Velasco, Cuba. ${ }^{2}$ Universidad de Holguín, Departamento de Física / Departamento de Química, Holguín, Cuba. ${ }^{3}$ Universidad de Oriente, Departamento de Química, Santiago de Cuba, Cuba.

*jvelazquezi@ueica.hlg.minag.cu

$\mathrm{R}$ esumen

Se estudió el efecto producido por las condiciones de transformación en la viscosidad del polietilentereftalato reciclado. Para ello, se realizó una simulación del procesado en un plastómetro para la determinación del Índice de Fluidez, sobre la base de un diseño de experimentos factorial $2^{2}$. Como variables independientes se tomaron la temperatura y tiempo de retención del polímero dentro de la cámara de procesamiento, mientras que como variable respuesta se evaluó la viscosidad intrínseca del polietilentereftalato. Mediante un análisis de regresión lineal del diseño experimental se logró obtener por primera vez la ecuación del modelo ajustado para la viscosidad intrínseca, sobre la que los dos factores estudiados (tiempo y temperatura) así como la interacción entre ellos presentaron un efecto significativo con un nivel de confianza de $95 \%$. En todos los casos ocurrió una disminución de la viscosidad del polietilentereftalato reciclado, encontrándose la mayor incidencia con un $30 \%$ al incrementar la temperatura de 260 a $280{ }^{\circ} \mathrm{C}$. De cara a un proceso de reciclado, se recomienda mantener un perfil de temperaturas que no supere los $260^{\circ} \mathrm{C}$ y tiempos de residencia igual o menores a $10 \mathrm{~min}$.

Palabras claves: Polietilentereftalato, reciclado, degradación química, viscosidad intrínseca, plastómetro.

A bstract

The effect produced by the processing conditions on the viscosity of recycled polyethylene terephthalate was studied. For this, a simulation of the processing was carried out using a melt flow index plastometer, based on a factorial design of experiments $2^{2}$. The temperature and the retention time of the polymer inside the processing chamber were selected as independent factors, while the intrinsic viscosity of the polyethylene terephtalate was the response variable. Through a linear regression analysis of the experimental design, it was possible to obtain for the first time the equation of the model adjusted for intrinsic viscosity, on which the two factors studied (time and temperature) as well as the interaction between them presented a significant effect with a level $95 \%$ confidence. In all cases, there was a decrease in the viscosity of the recycled polyethylene terephthalate, with the highest incidence being $30 \%$ when the temperature increased from 260 to $280^{\circ} \mathrm{C}$. For a recycling process, it is recommended to maintain a temperature profile that does not exceed $260{ }^{\circ} \mathrm{C}$ and residence times equal to or less than $10 \mathrm{~min}$.

Keywords: Polyethylene terephthalate, recycling, chemical degradation, intrinsic viscosity, plastometer. 


\section{INTRODUCCIÓN}

Los plásticos juegan un papel decisivo en la modernidad, estando presentes en prácticamente la totalidad de los campos de la vida del hombre (1-5). De hecho, el consumo global de plásticos en 2018 llegó a los 359 millones de toneladas métricas (6). Particularmente, la producción de polietilentereftalato (PET) en el mundo en 2016 llegó a los 50.01 millones de toneladas métricas, estimándose un ascenso hasta los $87.16 \mathrm{mi}-$ llones de toneladas métricas para 2022 (7). Por sectores de mercado, el PET se ha convertido en el material de envase más usado a nivel mundial para las botellas de agua y refrescos, representando esta aplicación el 83-84 \% del volumen consumido de PET en 2018 (8).

Estos objetos, debido a su corta vida de servicio (normalmente inferior a un año desde su manufactura), pasan rápidamente a formar parte de la corriente de desechos sólidos. La principal desventaja de los desechos plásticos proviene del elevado volumen que ocupan y de su extremadamente lenta velocidad de degradación, lo que conduce a su acumulación. Un ejemplo alarmante de esta situación es la enorme marea de desechos plásticos que flotan en los océanos, ocasionando graves daños a los ecosistemas (9). La revalorización de los desechos plásticos, es decir el reciclado y la recuperación de energía, constituyen importantes alternativas para darle otra vida a estos materiales $y$, al mismo tiempo, mitigar su impacto ambiental (10). Así, en las últimas dos décadas se ha incrementado el interés por el reciclado de los desechos plásticos, y especialmente el del polietilentereftalato. De hecho, el reciclado del PET representa uno de los ejemplos más exitosos y difundidos del proceso de reciclaje de polímeros (11).

La recuperación del PET se realiza predominantemente mediante el reciclado mecánico (12). Este es un proceso rápido y de un coste moderado, que permite la completa recuperación del PET y su utilización para fabricar nuevos productos dirigidos a varias aplicaciones industriales tales como el envasado de detergentes, cosméticos, alfombras, láminas, partes de autos o en nuevas botellas plásticas.
Sin embargo, en todos los casos la aplicación del polietilentereftalato reciclado dependerá fundamentalmente de las propiedades de la resina luego de su procesado. De hecho, la mayor dificultad enfrentada por todos los transformadores durante el reciclado del PET es su degradación. El PET es un poliéster alifático-aromático termoplástico semicristalino obtenido mediante reacciones de policondensación a partir de etilenglicol y ácido tereftálico o dimetiltereftalato (13). Por esta razón, al igual que ocurre para otros poliésteres orgánicos, su reacción de obtención es reversible y puede ocurrir la despolimerización, la que es acelerada por la presencia de agua, ácidos y/o por la temperatura (14). El efecto final se caracteriza por una acentuada pérdida de propiedades del polietilentereftalato reciclado. Por tanto, el control de la degradación del PET durante los procesos de transformación constituye la clave para obtener un producto de buenas prestaciones.

Varios investigadores han centrado su atención en los efectos del proceso de transformación en el PET. Sheldon (15) analizó la influencia de las condiciones de extrusión (velocidad de rotación/temperatura) en la cristalización del PET, encontrando que a elevadas velocidades de extrusión existe una tendencia a degradar las cristalitas u otras fuentes de nucleación, lo que afectó la velocidad de cristalización durante un posterior calentamiento. Curtzwiler y colaboradores (16) prepararon varias láminas para estudiar la influencia del contenido de PET reciclado (de 0 a $100 \%$ ) en las propiedades finales. Los ensayos mecánicos demostraron que el contenido de PET reciclado incorporado altera significativamente las propiedades de los materiales estudiados cuando eran comparados con la resina virgen. Giraldi y colaboradores (17) estudiaron nanocomposites de PET reciclado con contenidos de 2.5 y $5 \%$ de montmorillonita organomodificada, obtenidos mediante mezclado en fundido en una extrusora de doble husillos corrotantes a 250 y $150 \mathrm{rpm}$. Sobre la base de la influencia tiempo de residencia, encontraron que la velocidad de rotación de husillo puede optimizarse para conducir a la completa exfoliación de la nanoarcilla y, con esto, obtener las mejores propiedades mecánicas. Sin embargo, 
ninguno de ellos aborda los efectos sobre la estructura molecular del PET.

Por otra parte, Matayabas y colaboradores (18) al estudiar nanocomposites de PET con una organoarcilla comercial (Claytone APA), extruidos a $280^{\circ} \mathrm{C}$, encontraron una disminución de la viscosidad del PET, lo que se intensificó con el aumento del contenido de arcilla. Un resultado similar fue encontrado por Fang y colaboradores (19) cuando analizaron la influencia del contenido de carbonato de calcio $\left(\mathrm{CaCO}_{3}\right)$ y los parámetros de extrusión en el comportamiento de un masterbach y en fundido del PET reciclado. Al aumentar tanto el contenido de $\mathrm{CaCO}_{3}$ como la temperatura de la cámara de la extrusora de 240 a $260{ }^{\circ} \mathrm{C}$ ocurrió una disminución de la viscosidad intrínseca. No obstante, en ambos casos la presencia de los rellenos inorgánicos no deja claro cuál es la relación entre la viscosidad del PET y las condiciones de transformación.

El presente trabajo comprende el empleo de un plastómetro para realizar una simulación de un proceso de extrusión, como vía para el estudio de la influencia de las condiciones de transformación en la viscosidad del polietilenterftalato (PET) procedente de botellas plásticas de desecho.

\section{MATERIALES Y MÉTODOS}

Durante la investigación se empleó acetona (MercK, Alemania), éter (AnalaR, Inglaterra) y fenol (AnalaR, Inglaterra), todos con un $99 \%$ de pureza. El fenol fue destilado antes ser usado, mientras que el éter y la acetona se utilizaron tal y como se recibieron. El PET empleado se tomó de la reserva de botellas plásticas de desecho almacenada en la Empresa de Recuperación de Materias Primas de Holguín. A las botellas se les retiraron las tapas, etiquetas y demás materiales extraños. Por su parte, los restos de pegamento fueron cuidadosamente eliminados utilizando acetona, éter o agua. Seguidamente, se procedió al lavado, secado preliminar en estufa Electrothermal (Inglaterra) $\left(80^{\circ} \mathrm{C} / 1 \mathrm{~h}\right)$ y molido de las botellas en un molino desfibrador Nossen 8255 (Alemania). Las escamas de PET se sometieron a un tamizado, tomando para los ensayos la fracción que permaneció en el tamiz de $2 \mathrm{~mm}$. Finalmente, se realizó un secado de las escamas de PET a vacío a $100{ }^{\circ} \mathrm{C}$ durante 24 h en una estufa Memmert (Alemania), denominado PET-R. Además, con fines comparativos, se usó granza virgen de PET (PET-G) de la firma Intercontinental Polymers $\left([\eta]=0.635-0.665 \mathrm{dL} / \mathrm{g} \mathrm{y} \mathrm{T}_{\mathrm{f}}=\right.$ $\left.255^{\circ} \mathrm{C}\right)(20)$.

\section{Simulación del proceso de extrusión}

Para evaluar los posibles efectos derivados de la degradación ocurrida durante las etapas de extrusión del PET-R, se realizó una simulación del procesado en un plastómetro para la determinación del Índice de Fluidez (Ceast, Italia). Los experimentos se realizaron sobre la base de un diseño experimental factorial 22 , como vía para obtener la mayor cantidad de información con el menor número de experimentos posible (21). Como variables independientes se tomaron la temperatura y el tiempo de retención del polímero dentro de la cámara de procesamiento, mientras que como variable respuesta se tomó la viscosidad intrínseca [ $\eta]$. Se realizó una réplica, para un total de 8 experimentos. La tabla 1 muestra el diseño experimental completo utilizado. El rango de temperatura estudiado corresponde a la ventana de procesamiento utilizada por los transformadores del PET, mientras que el rango de tiempo de retención se basó en un estudio previo (22). Por su parte, el procesamiento estadístico se realizó utilizando el software "STATGRAPH”.

\begin{tabular}{ccc}
\hline Experimento & $\begin{array}{c}\text { Tiempo de retención }(\mathrm{t}) \\
(\mathbf{m i n})\end{array}$ & $\begin{array}{c}\text { Temperatura }(\mathrm{T}) \\
\left({ }^{\circ} \mathrm{C}\right)\end{array}$ \\
\hline E1 & 10 & 260 \\
E2 & 30 & 260 \\
E3 & 10 & 280 \\
E4 & 30 & 280 \\
\hline
\end{tabular}

Tabla 1. Diseño de los experimentos.

\section{Viscosimetría capilar}

Las medidas se efectuaron en un viscosímetro capilar Cannon Ubbelhode, termostatizado a la temperatura de $50 \pm 0.1^{\circ} \mathrm{C}$ en un baño de agua. Como disolvente se utilizó fenol. Se preparó una disolución madre de una concentración aproximada de $0.5 \mathrm{~g} / 100 \mathrm{~mL}$ por cada corrida experimental. Para ello, se pesaron aproximadamente $0.5 \mathrm{~g}$ del polímero y se colocaron en un vaso de precipitados al que se le adicionaron $100 \mathrm{~mL}$ de fenol. El beaker fue sellado y se colocó en un baño 
termostático (Bunsen, España) a $80{ }^{\circ} \mathrm{C}$ durante $1 \mathrm{~h}$, realizando agitaciones periódicas hasta completa disolución. De esta disolución, se tomaron $10 \mathrm{~mL}$ y se vertieron en el viscosímetro Cannon Ubbelhode, dejando transcurrir $30 \mathrm{~min}$. para que la solución se equilibrara a la temperatura de ensayo. El resto de disoluciones se obtuvieron in situ directamente en el viscosímetro, realizando adiciones sucesivas de disolvente puro. Seguidamente, se midieron los tiempos de flujo que tarda cada disolución y del disolvente puro en pasar entre dos marcas situadas en el viscosímetro. La relación entre el tiempo de caída de la disolución y del disolvente puro corresponde a la viscosidad relativa $\left(\eta_{\mathrm{r}}\right)$. Por su parte, la viscosidad específica se obtuvo restando uno a $\eta_{\mathrm{r}}$.

\section{RESULTADOS Y DISCUSIÓN}

Para la determinación de viscosidad intrínseca [ $\rceil$ de cada muestra se usaron las ecuaciones de Huggins (23) y Kraemer (24), ecuaciones (1) y (2) respectivamente. Se ha encontrado que estas ecuaciones son válidas a bajas concentraciones y la extrapolación gráfica de cualquiera de ellas a concentración igual a cero proporcione el mismo valor o valores muy aproximados de [ $\eta]$.

$$
\begin{aligned}
& \frac{\eta_{s p}}{c}=[\eta]+k_{H}[\eta]^{2} c \\
& \frac{\ln \eta_{r}}{c}=[\eta]-k_{K}[\eta]^{2} c
\end{aligned}
$$

Donde, $\eta_{\mathrm{sp}}$ es la viscosidad específica y $\mathrm{k}_{\mathrm{H}}$ y $\mathrm{k}_{\mathrm{K}}$ son las constantes de viscosidad de Huggins y Kraemer. En ambos casos, se requiere la determinación de sus viscosidades relativas $\left(\eta_{\mathrm{r}}\right)$ de una serie de soluciones, base para el cálculo de $\eta_{\mathrm{sp}} / c$, la cual es extrapolada a concentración cero para obtener a $[\eta]$. En este procedimiento se consume una gran cantidad de tiempo, esfuerzo y materiales. Por esta razón, varios autores han propuesto ecuaciones alternativas que permiten encontrar $[\eta]$ a partir de una sola determinación para una concentración conocida del polímero. Entre estos, Solomon y Ciuta (25) derivaron una relación para la determinación de $[\eta]$ a partir de un solo punto a partir de la combinación de las ecuaciones (1) y (2):

$$
[\eta]=\frac{1}{c} \sqrt{2\left(\eta_{s p}-\ln \eta_{r}\right)}
$$

La aplicabilidad de la ecuación (3) ha sido estudiada por varios investigadores. Elliot y colabo- radores (26) la emplearon para determinar [ $\eta]$ de muestras comerciales y experimentales de polipropileno y polietileno en decalina. Por su parte, Abdel-Azim y colaboradores (27) la usaron para estudiar varias muestras de polióxido de etileno y de polietilenglicol, así como poliestireno, utilizando benceno como disolvente. En ambos casos, se encontró que la ecuación de Solomon y Ciuta (ecuación 3) proporcionó valores aceptables de $[\eta]$.

Los valores de $[\eta]$ obtenidos para todas las corridas experimentales, empleando las ecuaciones (1) a la (3) se muestran en la tabla 2. Como puede apreciarse en los datos de la tabla 2, los valores de [ๆ] determinados según la extrapolación gráfica de Huggins y de Kraemer se encuentran en buen acuerdo, si bien los últimos son ligeramente superiores que los primeros. Por su parte, el uso de la ecuación (3) conjuntamente con el método clásico de la determinación en, al menos, tres puntos y la extrapolación gráfica se consideran suficientes para establecer si la ecuación de $\mathrm{Hu}$ ggins es aplicable al sistema polímero-disolvente

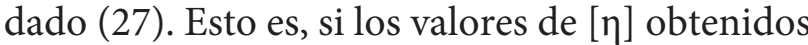
por extrapolación gráfica concuerdan con la [ $\eta]$ obtenida por la ecuación (3) entonces la relación de Huggins es aplicable. En este caso, independientemente de las condiciones experimentales, los valores de $[\eta]$ obtenidos de las ecuaciones propuestas por Huggins y por Solomon y Ciuta (Tabla 2) concuerdan. Esto le brinda soporte a

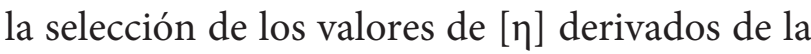

\begin{tabular}{|c|c|c|c|c|c|c|}
\hline \multirow{2}{*}{ Exp. } & \multicolumn{2}{|c|}{ Nivel } & \multirow[b]{2}{*}{$\eta_{\mathrm{sp}}$} & \multicolumn{3}{|c|}{$[\eta](\mathrm{cps})$} \\
\hline & $\mathrm{t}$ & $\mathrm{T}$ & & Huggins & Kraemer & $\begin{array}{c}\text { Solomon\&- } \\
\text { Ciuta }^{\text {a }} \\
\end{array}$ \\
\hline E1 & $--^{c}$ & - & $0.27 / 0.21 / 0.18 / 0.15 / 0.13$ & 0.4206 & 0.4203 & 0.4226 \\
\hline E2 & $+^{\mathrm{d}}$ & - & $0.26 / 0.20 / 0.16 / 0.14 / 0.12$ & 0.3479 & 0.3547 & 0.3817 \\
\hline E3 & - & + & $0.21 / 0.16 / 0.12 / 0.10 / 0.09$ & 0.2666 & 0.2733 & 0.2952 \\
\hline $\mathrm{E} 4$ & + & + & $0.22 / 0.16 / 0.13 / 0.11 / 0.09$ & 0.2577 & 0.2659 & 0.2631 \\
\hline $\mathrm{E} 1^{\mathrm{b}}$ & - & - & $0.27 / 0.21 / 0.17 / 0.16 / 0.12$ & 0.4120 & 0.4117 & 0.4139 \\
\hline $\mathrm{E} 2^{\mathrm{b}}$ & + & - & $0.25 / 0.20 / 0.16 / 0.13 / 0.12$ & 0.3599 & 0.3633 & 0.3752 \\
\hline $\mathrm{E} 3^{\mathrm{b}}$ & - & + & $0.24 / 0.18 / 0.15 / 0.12 / 0.10$ & 0.2823 & 0.2906 & 0.2879 \\
\hline $\mathrm{E} 4^{\mathrm{b}}$ & + & + & $0.25 / 0.18 / 0.15 / 0.12 / 0.09$ & 0.2525 & 0.2611 & 0.2581 \\
\hline
\end{tabular}
ecuación de Huggins para el análisis del diseño experimental seguido en esta investigación.

Tabla 2. Datos del diseño experimental $2^{2}$ elaborado para el estudio del efecto producido por los factores tiempo de retención y temperatura. ${ }^{\text {a }}$ Promedio de los valores de [ $\left.\eta\right]$ determinados para cada una de las 5 disoluciones evaluadas por muestra experimental.

${ }^{\mathrm{b}}$ Réplica.

${ }^{\mathrm{N}}$ Nivel mínimo.

${ }^{\mathrm{d}}$ Nivel máximo 
Mediante un análisis de regresión lineal aplicando el software de procesamiento estadístico STATGRAPH se obtuvo la siguiente ecuación del modelo ajustado:

$[\eta]=0.325875-0.058025 \mathrm{~A}-0.02015 \mathrm{~B}+0.01185 \mathrm{AB}$

Donde A corresponde a los valores de temperatura en ${ }^{\circ} \mathrm{C}, \mathrm{B}$ al tiempo en minutos y la multiplicación $\mathrm{AB}$ corresponde a la interacción de ambos factores.

Por su parte, la tabla 3 muestra el análisis de varianza (ANOVA) para [ $\eta$. En ella se particiona la variabilidad de $[\eta]$ en piezas separadas para cada uno de los efectos y se prueba su significancia estadística. Todos los efectos que tengan un valor $\mathrm{P}$ inferior a 0.05 son estadísticamente significativos con un nivel de confianza de un $95 \%$. En este caso, el tiempo, la temperatura así como la interacción entre ambos factores ejercen un efecto significativo sobre [ $\eta$ ]. Además, el elevado valor encontrado del coeficiente $\mathrm{R}^{2}$ de un 99.44 $\%$ indica que el modelo explica satisfactoriamente la variabilidad observada de [ $\eta$.

\begin{tabular}{llclrr}
\hline \multicolumn{1}{c}{ Fuente } & Suma de Cuadrados & Gl & Cuadrado Medio & Razón-F & Valor-P \\
\hline A: Temperatura & 0.026473 & 1 & 0.026473 & 463.44 & 0.0002 \\
B: Tiempo & 0.00271585 & 1 & 0.00271585 & 47.54 & 0.0063 \\
AB & 0.00104882 & 1 & 0.00104882 & 18.36 & 0.0234 \\
Bloques & 0.00006728 & 1 & 0.00006728 & 1.18 & 0.3572 \\
Error total & 0.00017137 & 3 & 0.0000571233 & & \\
Total (corr.) & 0.0304763 & 7 & & & \\
\hline
\end{tabular}

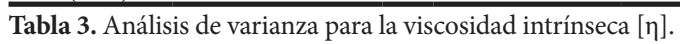

Para evaluar la influencia de cada factor estudiado en el diseño experimental se realizó la comparación de dos experimentos donde el segundo factor permaneció constante (Tabla 2). Así, la disminución de $[\eta]$ ocasionada por el incremento de la temperatura fue del orden del $30 \%$, mientras que el incremento del tiempo tuvo un efecto variable. A la temperatura de $260{ }^{\circ} \mathrm{C}$, se observó una disminución de [ $\eta$ ] de un $15 \%$ con el incremento del tiempo de residencia. Sin embargo, a $280^{\circ} \mathrm{C}$ el mismo incremento de tiempo sólo ocasionó una disminución del $7 \%$ de [ๆ].

Por su parte, en la figura 1 se representan los valores de $[\eta]$ para cada muestra experimental, así como también las viscosidades determinadas a muestras de granza virgen de PET (PET-G) y de las escamas de botellas de PET (PET-R) antes de ser introducidas en la cámara del plastómetro de Índice de Fluidez.

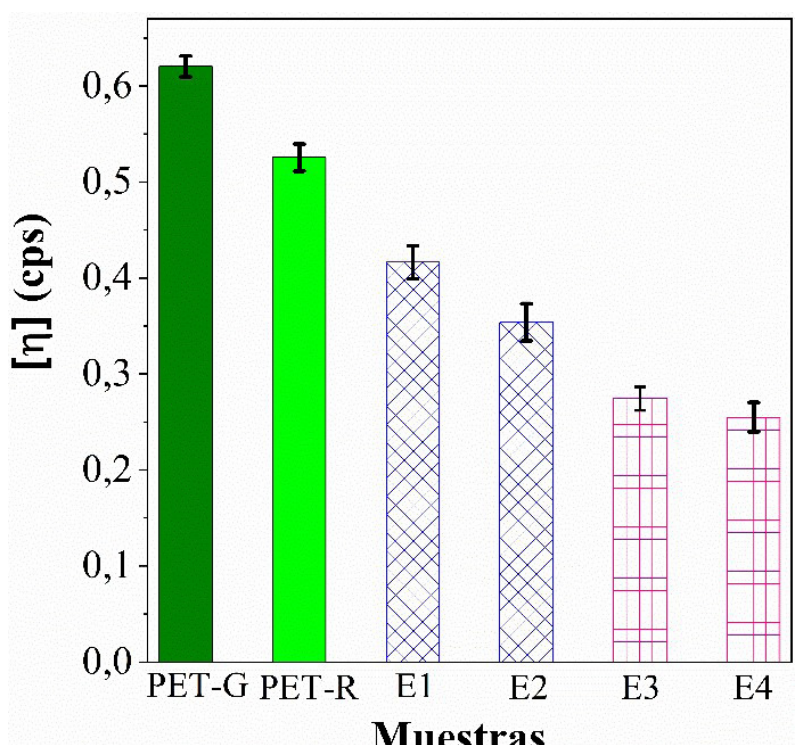

Figura 1. Valores de [ $\eta$ ] determinados mediante Huggins para muestras de los experimentos 1 al 4, de granza virgen de PET (PET-G) y de las escamas de botellas de PET (PET-R).

En todos los casos, la viscosidad intrínseca de las muestras de PET que han experimentado, al menos, una etapa de procesado fue menor que la de la granza virgen (Fig. 1). Así mismo, al comparar las muestras de los experimentos 1 al 4 (todos con 2 etapas de procesado) con el PET-R ( 1 etapa de procesado), se puede apreciar que el incremento del número de etapas de procesa-

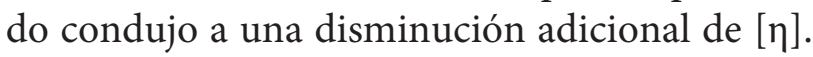
De acuerdo con la ecuación de Mark-Houwink (Ecuación 4), existe una relación directa entre la viscosidad intrínseca y la masa molecular de un polímero. Por tanto, esta disminución observada de $[\eta]$ estaría indicando una reducción significativa del tamaño molecular de las cadenas de PET, trayendo consigo un deterioro de las propiedades mecánicas del material reciclado (28).

$$
[\eta]=K \times M_{v}^{a}
$$

Esta reducción del peso molecular puede tener lugar durante la transformación del PET, fundamentalmente, mediante procesos de degradación térmica, hidrolítica y/o termo-oxidativa. Las reacciones de degradación térmica e hidrolítica se han estudiado extensivamente (29-31). La degradación térmica ocurre mediante un proceso iónico, en el cual, inicialmente, ocurre la transferencia de un hidrógeno $\beta-\mathrm{CH}$ seguido por la ruptura de la cadena y el origen de compuestos vinílicos y carboxílicos. También, puede ocurrir como consecuencia de un proceso de re-arreglo molecular generando productos oligoméricos cíclicos. Por su parte, las reacciones de hidrólisis 
originan grupos terminales carboxilo e hidroxilo. Algunos investigadores (29) la han catalogado como un mecanismo auto-catalítico, potenciado por el incremento en el número de grupos carboxilo terminales. Esta es una reacción extremadamente sensible (32) y tiene lugar a partir de un contenido de agua ligeramente superior a $0 \mathrm{ppm}$.

En el caso de la degradación térmica oxidativa, se considera que se produce por la reacción del oxígeno con el PET a elevadas temperaturas (31). El mecanismo exacto de la degradación oxidativa del PET es muy complejo y no está claro, pero se ha propuesto que transcurre por medio de radicales libres. El paso inicial de este mecanismo, conduce a la formación de hidroperóxidos en los enlaces del dietilenglicol de las cadenas del polímero. Estudios demuestran que las unidades de dietilenglicol son particularmente reactivas y se combinan con el oxígeno para formar hidroperóxidos (temperaturas por debajo de 200 $\left.{ }^{\circ} \mathrm{C}\right)$ (33). A temperaturas mayores $\left(\sim T_{f}\right)$; estos se descomponen dando lugar a una serie de reacciones que concluyen con la ruptura de la cadena. Sin embargo, este no es el único efecto ya que también traen consigo la ramificación de las cadenas y la formación del dióxido de carbono $\left(\mathrm{CO}_{2}\right)$. Estos últimos efectos se explican por la descarboxilación del radical carboxilo seguida por la adición del grupo fenilo resultante a otro anillo aromático, como se muestra en la figura 2 . De ese modo, en las muestras procesadas a 280 ${ }^{\circ} \mathrm{C}$ podría estar ocurriendo entrecruzamiento, lo que traería consigo un incremento de la viscosidad del PET, enmascarando la disminución de $[\eta]$ derivada de las reacciones de escisión de cadena.

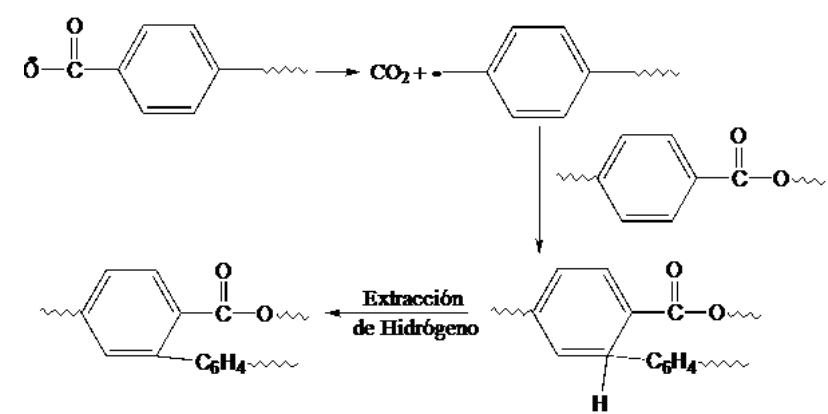

Figura 2. Reacciones de ramificación de las cadenas y formación del dióxido de carbono [Adaptado de (13)].

\section{CONCLUSIONES}

Se estudió la influencia de las condiciones de procesamiento en el polietilentereftalato reciclado, obteniéndose por primera vez la ecuación del modelo ajustado por regresión lineal para la viscosidad intrínseca [ $\eta$ ]. Los factores estudiados, temperatura de procesamiento y tiempo de residencia, así como su interacción presentaron dentro del rango estudiado un efecto significativo con un $95 \%$ de confianza. Si bien en todas las corridas experimentales se observó una disminución de la viscosidad, indicando una reducción del tamaño molecular, el incremento de la temperatura ocasionó la mayor reducción de $[\eta]$ en aproximadamente un $30 \%$.

Así, la determinación de [ๆ] constituye una vía útil para optimizar el proceso de reciclaje del PET. De cara a un proceso de reciclado, para minimizar los procesos de degradación durante su transformación en fundido y el consecuente deterioro de las propiedades del PET, se recomienda mantener un perfil de temperaturas que no supere los $260^{\circ} \mathrm{C}$ así como mantener un tiempo de residencia igual o menor a $10 \mathrm{~min}$.

$\mathrm{R}$ eferencias

1. Çaykara T, Sande MG, Azoia N, Rodrigues LR and Silva CJ. Exploring the potential of polyethylene terephthalate in the design of antibacterial surfaces. Med. Microb. and Imm. 2020; 209: 363-372.

2. Begum SA, Rane AV and Kanny K. Applications of compatibilized polymer blends in automobile industry. En: Ajitha AR and Sabu Thomas, Editores. Compatibilization of Polymer Blends. Micro and Nano Scale Phase Morphologies, Interphase Characterization and Properties. eBook. Amsterdam: Elsevier, 2020. p. 563-593.

3. Huang J, Yang X, Yu J, Han J, Jia C, Ding M et al. A Universal and Arbitrary Tactile Interactive System Based on Self-powered Optical Communication. Nan. Energ. 2020; 69 (March): 10441. 
4. Zeng JJ, Gao WY, Duan ZJ, Bai YL, Guo YC, Ouyang LJ. Axial compressive behavior of polyethylene terephthalate/carbon FRP confined seawater sea-sand concrete in circular columns. Cons. and Build. Mat. 2020; 234: 117383.

5. Senthil Kumar P and Yaashikaa PR. Case Studies on Recycled Polyesters and Different Applications. En: Subramanian Senthilkannan Muthu, editor. Environmental Footprints of Recycled Polyester. eBook. Singapore: Springer; 2020. p. 99-112.

6. Scribd Inc. Plásticos-Situación en 2019 [Internet]. 1ra ed. California: Trip Adler; 2020 [actualizado 20 Septiembre 2021; citado 15 Agosto 2021]. Disponible en: https://es.scribd.com/ document/484313664/Plastics-the-facts-Mar2019-esp.

7. Business wire Inc. Global Polyethylene Terephtalate Market Report 2017 - By End-Use Industries, Products \& Regions - Research and Markets [Internet]. Dublin: Business Wire; 2017 [actualizado 10 Enero 2021; citado 12 Agosto 2021]. Disponible en: https://www.businesswire.com/news/home/20170914005775/en/Global-Polyethylene-Terephtalate-Market-Report2017---By-End-Use-Industries-Products-Regions---Research-and-Markets.

8. Zhang $\mathrm{H}$ and Wen ZG. The consumption and recycling collection system of PET bottles: a case study of Beijing, China. Wast. Manag. 2014; 34: 987-998.

9. Sasse, F and Emig, G. Chemical recycling of polymer materials. Chem. Eng. Technol. 1998; 21: 777-789.

10. Stanica-Ezeanu D and Matei D. Natural depolymerization of waste poly(ethylene terephthalate) by neutral hydrolysis in marine water. Sci. Rep. 2021; 11: 4431.

11. Throne JL. Effect of recycle on properties and profits: Algorithms. Adv. Polym. Tech. 1987; 7: 347-360.

12. Gupta VB, Bashir Z. PET Fibers, Films, and Bottles: Sections 1-4. En: Fakirov S, editor. Handbook of Thermoplastic Polyesters. Vol 1. 2da ed. Weinheim: Wiley VCH Verlag GmbH \& Co. KGaA, 2002. p. 317-361.

13. Brandrup J. Polymer handbook. Vol 2. 4ta ed. New York: Intersciene Publishers; 1978.

14. Silva Freitas FL, Chinellato AC, Rodrigues Pereira Filho E and Cruz SA. Evaluation of the effect of additives on thermo-oxidative and hydrolytic stabilization of recycled post-consumer poly (ethylene terephthalate) using Design of Experiments. Polym. Test. 2020; 81: 106275. 15. Sheldon, RP. The influence of extrusion conditions on the crystallization of polyethylene terephthalate film. Polym. 1963; 4: 213-219.

16. Curtzwiler G, Vorst K, Danes JE, Auras R, Singh J. Effect of recycled poly(ethylene terephthalate) content on properties of extruded poly(ethylene terephthalate) sheets. J. of Plast. Film \& Sheet. 2011; 27: 65-86.

17. Giraldi ALFM, Bizarria MTM, Silva AA, Velasco JI, Marcos AdA, Mei LHI. Effects of Extrusion Conditions on the Properties of Recycled Poly(Ethylene Terephthalate)/Nanoclay Nanocomposites Prepared by a Twin-Screw Extruder. J. of App. Polym. Sci. 2008; 108: 22522259.

18. Matayabas JC, Turner SR. Nanocomposite technology for enhancing the gas barrier of polyethylene terephtalate. In: Pinnavaia TJ and Beall GW, editores. Polymer-clay nanocomposites. Vol 1. 1ra ed. New York: John Wiley \& Sons Ltd; 2000. p. 207-225.

19. Fang T, Li J, Yan W, Gu D, Du Z. Effect of calcium carbonate as pore-forming agent on properties of recycled polyethylene terephthalate masterbatch. J. of Phys.: Conference Series. 2021; 1790: 012011.

20. Matweb Material Properties Data [Página principal de internet], Blacksburg: MatWeb LLC; 2020. [actualizada en Marzo de 2011; acceso 10 agosto 2021] http://www.matweb.com/ search/datasheet.aspx?matguid=6e09c609f7364fc6a997e6bbafc609d7\&ckck=1.

21. Lopez R. Diseño estadístico de experimentos. Vol 1. 3ra ed. Ciudad de La Habana: Científico-Técnica; 1988.

22. Velázquez-Infante JC, Pérez-Rodríguez AT y García JM. Estudio del proceso de recicla- 
do de las botellas de polietilentereftalato. En: Francisco Torres Editor. Libro de memorias. 6ta Convención y Feria Internacionales "METANICA 2001". 2001 Jul 17-20; La Habana. Ciudad de La Habana: Científico Técnica; 2001. 101-109.

23. Huggins ML. The Viscosity of Dilute Solutions of Long-Chain Molecules. IV. Dependence on Concentration. J. Am. Chem. Soc. 1942; 64: 2716-2718.

24. Kraemer EO. Molecular Weights of Celluloses and Cellulose Derivates. Ind. Eng. Chem. 1938; 30: 1200-1203.

25. Solomon OF and Ciuta IZ. Détermination de la viscosité intrinsèque de solutions de polymères par une simple détermination de la viscosité. J. Appl. Polym. Sci. 1962; 6: 683-686.

26. Elliott JH, Horowitz $\mathrm{KH}$ and Hoodock T. A one-point intrinsic viscosity method for polyethylene and polypropylene. J. Appl. Polym. Sci. 1970; 14: 2947-2963.

27. Abdel-Azim AA, Atta MA, Farahat MS and Boutros WY. Determination of intrinsic viscosity of polymeric compounds through a single specific viscosity measurement. Polym. 1998; 39: 6827-6833.

28. Masmoudi F, Alix S, Buet S, Mehri A, Bessadok A, Jaziri M et al. Design and Characterization of a New Food Packaging Material by Recycling Blends Virgin and Recovered polyethylene terephthalate. Polym. Eng. Sci. 2020; 60: 250-256.

29. Zimmerman H and Kim NT. Investigations on thermal and hydrolytic degradation of poly (ethylene terephthalate). Polym. Eng. Sci. 1980; 20: 680-683.

30. Housseini SS, Taheri S, Zadhoush A, Mehrabani-Zeinabad A. Hydrolytic degradation of poly (ethylene terephthalate). J. Appl. Polym. Sci. 2007; 103: 2304-2309.

31. Badia JD, Martinez-Felipe A, Santoja-Blasco L, Ribes-Greus A. Thermal and thermo-oxidative stability of reprocessed poly (ethylene terephthalate). J. Anal. Appl. Pyr. 2013; 99: 191-202. 32. Al-Abdulrazzak S, Jabarin SA. Processing characteristics of poly (ethylene terephthalate): hydrolytic and thermal degradation. Polym. Int. 2002; 51: 164-173.

33. Buxbaum LH. The Degradation of Poly (ethylene terephthalate). Ang. Chem. Int. 1968; 7: $182-190$. 